\title{
Utilization of Ultrasound for Management of Surgical Intervention of Secondary Hyperparathyroidism and Prolonged Hypocalcemia Post-Parathyroidectomy
}

\author{
Ying Liu, MM ${ }^{a, 1}$, Yang Zhou, MD ${ }^{a, 1}$, Hong Zhou, BS ${ }^{a, *}$, Yuanyuan Chen, MM ${ }^{b}$, Jian Wu, MD ${ }^{c}$, Juan Wang, BS ${ }^{a}$, \\ Bin Wang, MM ${ }^{c}$, Changyu Chen, $M^{a}{ }^{a}$, Ming Ye, MM ${ }^{a}$ \\ ${ }^{a}$ Department of Ultrasound, The Third People's Hospital of Chengdu, The Affiliated Hospital of Southwest Jiaotong University and the \\ Second Affiliated Clinical Hospital of Chongqing Medical University, Chengdu, China; ${ }^{b}$ Department of Pathology, The Third People's \\ Hospital of Chengdu, The Affiliated Hospital of Southwest Jiaotong University and the Second Affiliated Clinical Hospital of Chongqing \\ Medical University, Chengdu, China; ${ }^{c}$ Department of Thyroid and Breast Surgery, The Third People's Hospital of Chengdu, The Affiliated \\ Hospital of Southwest Jiaotong University and the Second Affiliated Clinical Hospital of Chongqing Medical University, Chengdu, China \\ Received September 15, 2020; revision received October 30, 2020; accepted November 08, 2020
}

Objective: To evaluate the application of ultrasound (US) for the surgical intervention in patients with moderate and severe secondary hyperparathyroidism (SHPT), and to identify the risk of prolonged hypocalcemia after parathyroidectomy (PTX).

Methods: A consecutive series of moderate and severe SHPT patients $(n=64)$ underwent ultrasound evaluation of parathyroid glands. Among the 64 patients who received 6-month medication therapies, ten patients with parathyroid hormone (PTH) 300 500 $\mathrm{pg} / \mathrm{mL}$ were excluded from the study while 32 patients unresponsive to medication therapy (PTH $>500 \mathrm{pg} / \mathrm{mL}$ ) received surgical interventions and 22 patients with PTH $<300 \mathrm{pg} / \mathrm{mL}$ received medication treatment alone. The correlations between the number, location, volume, sonographic features of parathyroid glands (PTGs), laboratory examinations, the duration of dialysis and the surgical necessity were analyzed. Total parathyroidectomy with synchronous auto-transplantation (PTX $+\mathrm{AT}$ ) was performed in the surgical group. In both the surgical and medication group, patients with hyper-vascularity of the PTGs dominated $(\geqslant 50 \%)$ were classified as a hyper-vascular subgroup, and the others as a hypo-vascular subgroup. The differences of post-operative calcium $\left(\mathrm{Ca}^{2+}\right)$ levels and the incidence of prolonged hypocalcemia between hyper- and hypo- vascular subgroups were assessed.

Results: Sonographic evaluations revealed that the numbers of detectable PTGs were higher in the surgical group than that of the medication group $(p<0.05)$. The detection of supernumerary PTGs was higher in the surgical group than that in the medication group $(13 / 121,10.7 \%$ vs. 2/71, 2.8\%, $p<0.05)$. Baseline PTH, $>2$ detectable PTGs, detection of supernumerary PTGs, patients with hyper-vascular, and the duration of dialysis were positively associated with the necessity of surgical intervention. For patients in the hyper-vascular subgroup, the serum $\mathrm{Ca}^{2+}$ level was lower than that in the hypo-vascularity subgroup $(p<0.01)$.

Conclusion: Ultrasonic features can provide useful information for management of surgical intervention of SHPT and prediction of the risk of prolonged hypocalcemia after PXT.

Key words: Secondary hyperparathyroidism; Ultrasound; Total parathyroidectomy; Auto transplantation; Hypocalcemia

Advanced Ultrasound in Diagnosis and Therapy 2021; 03: 236-244

DOI: 10.37015/AUDT.2021.200060

\footnotetext{
${ }^{1}$ Co-first author

* Corresponding author: Hong Zhou, Department of Ultrasound, The Third People's Hospital of Chengdu, The Affiliated Hospital of Southwest Jiaotong University and the Second Affiliated Clinical Hospital of Chongqing Medical University, Chengdu, China.

e-mail:664121529@qq.com
} unrestricted use, distribution and reproduction in any medium provided that the original work is properly attributed. 
$\mathrm{S}$ econdary hyperparathyroidism (SHPT) is caused by the hyperplasia of the parathyroid glands (PTGs) in patients with chronic renal failure (CKD) $[1,2]$. Severe SHPT is associated with increased cardiovascular mortality [3]. Hyperplasia of PTGs is only reversible at early stage [4]. The response of SHPT patients to pharmacological therapy fell short of expectations in the past few decades. New pharmacological therapies (calcitriol, active vitamin $\mathrm{D}$, and phosphate-binding drugs) significantly delay the surgery timeline for two years [5]. Nevertheless, surgical intervention, which is cost-saving, is still meaningful for the alleviation of symptoms in patients who fail to respond to medication therapy. Previous studies revealed that the sizes of PTGs and the level of parathyroid hormone $(\mathrm{PTH})$ could predict the patients' response to drugs, however, such studies included patients with mildly increased PTH, which could be managed medically [6-8].

Meanwhile, the cut-off value of PTH for aggressive intervention has not yet been established. The acceptable PTH values in the JSDT guidelines is $<240 \mathrm{pg} / \mathrm{mL}$, whereas those in the National Kidney Foundation Kidney Disease Outcomes Quality Initiative and the Kidney Disease Improving Global Outcomes guidelines are $<300 \mathrm{pg} / \mathrm{mL}$ and $<600 \mathrm{pg} / \mathrm{mL}$, respectively [79]. In clinical practice, patients were categorized according to the PTH values: mild $(<500 \mathrm{pg} / \mathrm{mL})$, moderate $(500-800 \mathrm{pg} / \mathrm{mL})$, and severe $(>800 \mathrm{pg} / \mathrm{mL})$. Mild patients more likely respond to pharmacological therapies [9]. At present, there are no clinical guidelines advocating a timely parathyroidectomy for moderate and severe patients. Ultrasonography, as a useful tool to define the size, structure, vascularity of PTGs even the type of hyperplasia, is rarely utilized to help clinicians make clinical decisions, such as whether the surgical intervention should be considered $[8,10]$.

Hungry bone syndrome (HBS), mainly manifested as prolonged hypocalcemia (serum $\mathrm{Ca}^{2+}<2.1 \mathrm{mmol} / \mathrm{L}$, lasting more than $4 \mathrm{~d}$ ), is a critical post-parathyroidectomy complication, with an incidence ranging from $27.8 \%$ to $72 \%$ [11]. It may cause laryngeal muscle spasm leading to asphyxia, cardiac dysfunction, or even cardiac arrest. The sudden decline of PTH leads to an enhanced bone uptake of $\mathrm{Ca}^{2+}$, phosphate (P) and magnesium $\left(\mathrm{Mg}^{2+}\right)$ with unaffected osteoblast activity, which results in an increase in bone density. Several risk factors have been identified to indicate the development of HBS, including age, size of resected parathyroid glands, preoperative serum PTH and alkaline phosphatase (ALP) level [12]. However, there is a paucity of data concerning the preoperative sonographic assessment to predict the prolonged hypocalcemia or even HBS in patients with SHPT.
Therefore, the aim of this was to evaluate the application of ultrasound (US) for the surgical intervention in patients with of moderate and severe secondary hyperparathyroidism (SHPT), and to identify the risk of prolonged hypocalcemia after parathyroidectomy (PTX). We analyzed the association between the PTGs' sonographic patterns and the necessity of surgeries, and predicting the risk for prolonged hypocalcemia after PXT accordingly.

\section{Materials and Methods}

\section{Study design and ethical approval}

Between January 2018 and July 2019, a consecutive series of 64 patients on maintenance bicarbonate hemodialysis at the dialysis unit of the Third People's Hospital of Chengdu had PTH levels $>500 \mathrm{pg} / \mathrm{mL}$ at admission and enrolled in this study. To reduce the PTH level in 6 months, 52 participants received combined administration of elemental calcium $\left(\mathrm{Ca}^{2+}\right) 1000 \mathrm{mg}$ to $1200 \mathrm{mg}$ and vitamin $\mathrm{D}_{3} 20 \mathrm{mg}$ (800 IU) daily, while the other 12 patients were prescribed calcimimetics. According to consecutive laboratory testing and clinical features within the previous 6 months, ten patients whose PTH levels were between $300 \mathrm{pg} / \mathrm{mL}$ and $500 \mathrm{pg} / \mathrm{mL}$ after treatments were excluded as the dubious curative effect of medication. Among the other 54 patients, the surgical group consisted of 32 patients unresponsive to medication therapy (defined as PTH $>500 \mathrm{pg} / \mathrm{mL}$ independent of serum $\mathrm{Ca}^{2+}$ level) who received surgical intervention. Surgical exclusion criteria included heart failure, severe pulmonary hypertension, and coagulation disorders. The medication group consisted of the remaining 22 patients with progressive decline of $\mathrm{PTH}$, defined as median PTH level $<300 \mathrm{pg} / \mathrm{mL}$ over three consecutive monthly measurements after the 6-months therapy [6], who had received pharmacological treatment.

At admission and 6 months after medication therapy, all patients received high-resolution US examination by two independent sonographers (both of which had more than 10 years experiences and performed a consistency test at the start of the study). An IU Elite scanner (Phillips Medical Systems, Bothell, WA, USA) equipped with an L12-5 transducer was used. The number, position, volume, sonographic structures and vascularity of detectable PTGs were recorded, including classical pairs of PTGs and all supernumerary PTGs. Standardized video documentation of ultrasonic scan from the bilateral lower jaw to the supraclavicular fossa was recorded, followed by standardized longitudinal and transverse views of each detectable PTG. In addition, the three dimensions of PTGs were measured. The PTG size was defined as the maximal longitudinal diameter (MLD), 
and the volume of each PTG was estimated as $\pi / 6 \times$ width $(\mathrm{mm}) \times$ length $(\mathrm{mm}) \times$ depth $(\mathrm{mm})[13]$. After that, the section with the most abundant blood flow of each PTG was recorded, while the vascularity was assessed and graded according to Doppler score Onoda [6]: (1) a few star or dot blood flow signals in surrounding glands presents Peripheral P1, (2) long streak signals surrounding more than $30 \%$ of PTG circumference presents Peripheral P2, (3) a few short or dot blood flow signals in the central region of glands presents Central $\mathrm{C} 1$, (4) long streak bloodstream in the inside of the gland ( $>30 \%$ of the surface) presents Central $\mathrm{C} 2$, and (5) no blood flow inside the gland presents Central C0. Since the central blood supply is more important than the peripheral flow, hypo-vascular PTGs were defined as scored P0/1/2, C0 while hyper-vascular PTGs as scored $\mathrm{P} 0 / 1 / 2, \mathrm{C} 1$ or $\mathrm{P} 0 / 1 / 2, \mathrm{C} 2$. Patients whose hypovascularity of the PTGs dominated $(\geqslant 50 \%)$ were categorized as the hypo-vascular subgroup (Fig. 1), while the others as the hyper-vascular subgroup (Fig. 2). Meanwhile, 32 patients in surgical group received preoperative ${ }^{99 \mathrm{~m}} \mathrm{Tc}-\mathrm{MIBI}$ SPECT/CT scans.

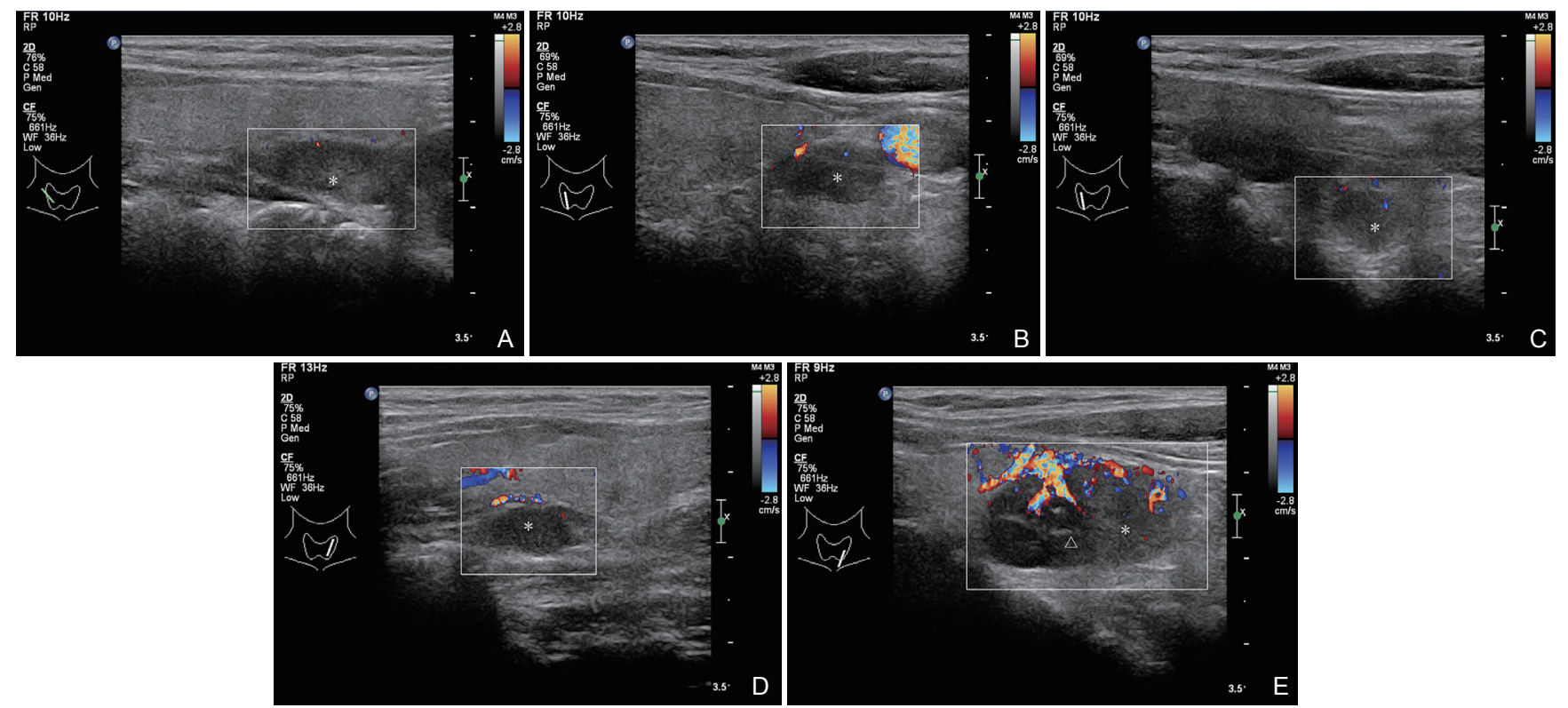

Figure 1 Sonographic images of PTGs in a 31 years old female patient in the hypo-vascular subgroup. There were five detectable PTGs, in which most of them (4/5) were hypo-vascular feature. (A). Longitudinal image of the gland in a right superior position (*), the gland was slightly heterogeneous, appearing to be partial hypoechoic and partial hyperechoic. The Doppler score Onoda was P1, C0; (B). Longitudinal image of the gland in the right inferior position (*), appearing to be hypoechoic with Doppler score Onoda P1, C0; (C). Longitudinal image of a supernumerary PTG in the right inferior position. This "newborn" echoic gland (*) was with Doppler score Onoda P1, C0; (D). Longitudinal image of a gland in a left superior position (*), appearing to be hypoechoic with the Doppler score Onoda P2, C0; (E). Longitudinal image of a gland in the left inferior position, appearing to be highly heterogeneous with partial hyperechoic $(\triangle)$ and partial hypoechoic. The Doppler score Onoda was P2, C2.

Total parathyroidectomy with synchronous autotransplantation (PTX + AT) was performed with careful exploration and intraoperative pathology to ensure thorough removal of all PTGs $[14,15]$. Pieces $(10-$ $20 \mathrm{~mm}^{3}$ ) minced from the most normal appearing gland were implanted into the forearm of each patient. All patients received a postoperative intravenous $\mathrm{Ca}^{2+}$ infusion at a rate of $2720 \mathrm{mg}$ elemental $\mathrm{Ca}^{2+} /$ day. If serum $\mathrm{Ca}^{2+}$ level was $2.1 \mathrm{mmol} / \mathrm{L}$ or lower, standard calcium dialysate was used. Additionally, an extra $10 \mathrm{~mL}$ of $10 \%$ calcium chloride solution $\left(272 \mathrm{mg}\right.$ elemental $\mathrm{Ca}^{2+}$ ) was given with monitoring calcium ions [16]. Serum $\mathrm{Ca}^{2+}$ and PTH levels were monitored every $6 \mathrm{~h}$ immediately after the surgery for a week, then every two days for another one week, after which, the laboratory tests were ordered biweekly during the 6-month follow-up. This study was approved by the Ethics Committee of the Third People's Hospital of
Chengdu (Sichuan, China), and all participants provided written informed consent.

\section{Statistical analysis}

The SPSS 22.0 software package (IBM, Armonk, NY, USA) was used for statistical analysis. Values were expressed as the mean $\pm \mathrm{SD}$. Continuous variables were compared between groups using the $t$-test. Chi square test was used to compare and analyze the frequencies. Multiple logistic regression analysis was used to analyze the association between sonographic features, laboratory examinations and the necessity of surgeries of SHPT. Statistical significance was considered as $p<0.05$.

\section{Results}

Sonographic evaluations revealed that the numbers of detectable PTGs were higher in the surgical group than 
that of the medication group $(p<0.05)$. In the surgical group, in addition to the classical pairs of superior and inferior PTGs, thirteen supernumerary PTGs were detected by US and confirmed by surgeries. In contrast, only two supernumerary PTGs located posterior to the thyroid gland were identified in the medication group. The detection of supernumerary PTGs was higher in the surgical group than that in the medication group $(13 / 121,10.7 \%$ vs. $2 / 71$, $2.8 \%, p<0.05)$. Compared with the medication group, the surgical group had a higher proportion of hyper-vascular subgroup patients $(p<0.05)$ and a lower proportion of hypo-vascular subgroup patients $(p<0.05)$. All ultrasonic features of PTGs in both groups remained unchanged after 6 months (Table 1).
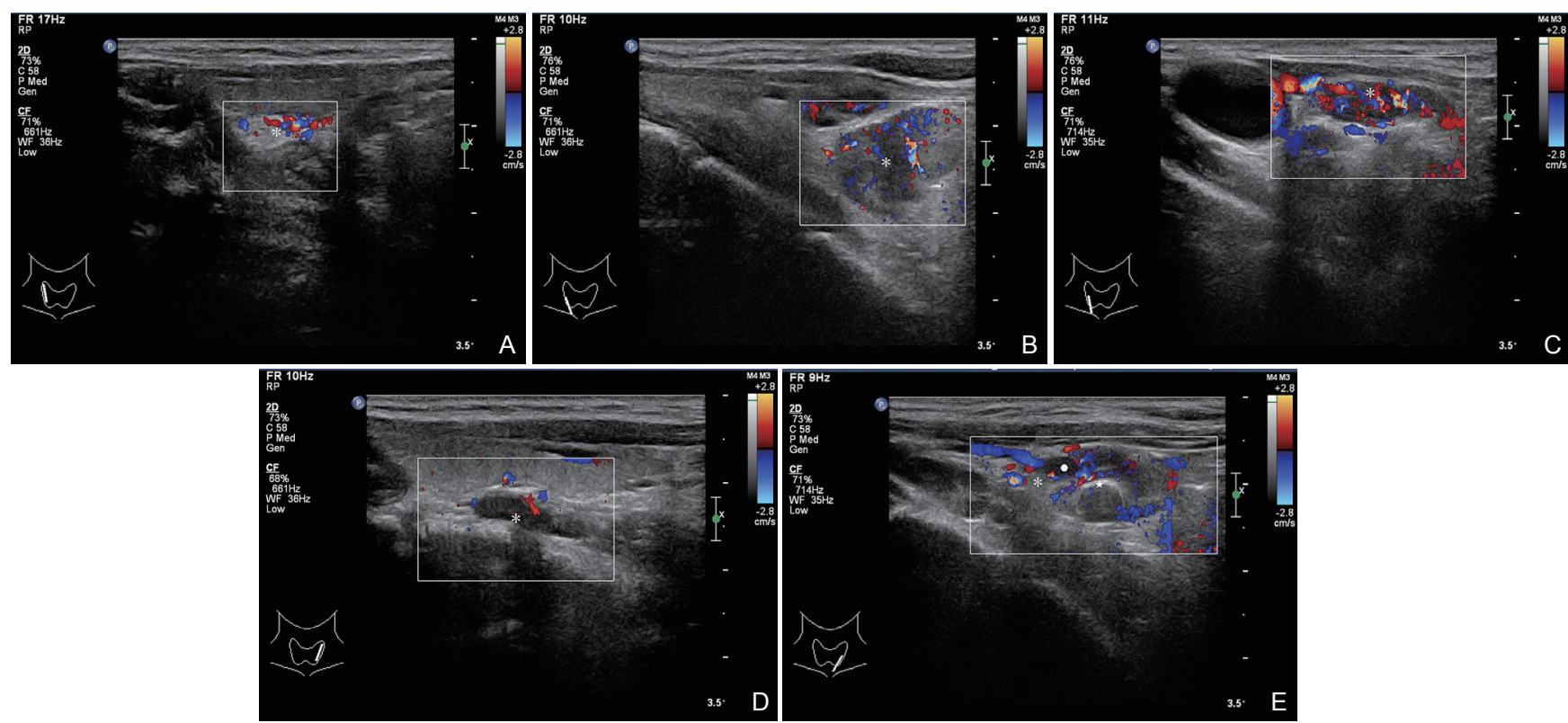

Figure 2 Sonographic images of PTGs of a 46 years old female patient in hyper-vascular subgroup. The total number of detectable PTGs was 5 . Most of these PTGs (4/5) were hyper-vascular. (A) The longitudinal image showed the gland in right superior position (*), which was heterogeneously hyperechoic with the Doppler score Onoda was P1, C2; (B) The longitudinal image showed the gland in the right inferior position $(*)$, which was medium heterogeneous, appearing to be partial hypoechoic and partial hyperechoic, The Doppler score Onoda was P2, C2;(C) The longitudinal image showed a supernumerary PTG gland $(*)$ in the right inferior position, which shown to be homogeneous hypoechoic with Doppler score Onoda P2, C2; (D) The longitudinal image showed a gland $(*)$ in the left superior position, which was slightly heterogeneous with Doppler score Onoda P1, C0; (E) The longitudinal image showed another gland (*) in the left inferior position, which was highly heterogeneous with annular calcification (white $\star$ ), cystic appearance (white circle) and Doppler score Onoda P1, C2.

Table 1 Patients with ultrasonic detective PTGs in surgical and medication group

\begin{tabular}{|lccc}
\hline Items & Numbers of patients & Numbers of PTGs in 4 classical positions & Numbers of supernumerary PTGs \\
\hline Surgical Group & 32 & 108 & 13 \\
\hline Patients with 1 US detectable PTG & 1 & 1 & 0 \\
\hline Patients with 2 US detectable PTGs & 4 & 8 & 0 \\
\hline Patients with 3 US detectable PTGs & 5 & 14 & 1 \\
\hline Patients with 4 US detectable PTGs & 16 & 61 & 3 \\
\hline Patients with 5 US detectable PTGs & 3 & 12 & 3 \\
\hline Patients with 6 US detectable PTGs & 3 & 12 & 6 \\
\hline Medication Group & 22 & 69 & 2 \\
\hline Patients with 1 US detectable PTG & 3 & 3 & 0 \\
\hline Patients with 2 US detectable PTGs & 10 & 20 & 1 \\
\hline Patients with 3 US detectable PTGs & 4 & 11 & 1 \\
\hline Patients with 4 US detectable PTGs & 5 & 19 & 0 \\
\hline Patients with 5 US detectable PTGs & 0 & 0 & 0 \\
\hline Patients with 6 US detectable PTGs & 0 & 0 & 2 \\
\hline
\end{tabular}

Chi square test was used to compare and analyze the frequencies. 
As shown in Table 2, serum $\mathrm{P}$, calcium-phosphorus product, baseline PTH (PTH at admission), duration of dialysis, the number, the average MLD and volume of US detectable PTGs were increased in the surgical group versus the medication group (all $p<0.05$, respectively). In contrast, the serum $\mathrm{Ca}^{2+}$ was lower in the surgical group compared to the medication group $(p<0.01)$. In the surgical group, 32 patients received PTX + AT due to poor response to pharmacological therapy after 6 months. A higher proportion of patients with $>2$ detectable PTGs was observed in the surgical group compared to the medication group $(84.4 \%$ vs. $40.9 \%, p<0.05)$. Between the two groups, there was no statistical significance found in the prevalence of PTGs with hyper-echogenicity, hypo-echogenicity, heterogeneity, calcification, or cystic degeneration, respectively. Multiple logistic regression analysis revealed that the baseline PTH (HR 95\% CI 3.5, $p<0.05$ ), $>2$ detectable PTGs (HR 95\%CI 1.8, $p<$ 0.05 ), detection of supernumerary PTGs (HR 95\%CI 1.2, $p<0.05$ ), patients of the hyper-vascular subgroup (HR $95 \%$ CI $1.5, p<0.05$ ), and the duration of dialysis (HR $95 \%$ CI $2.40, p<0.05$ ) were correlated with the necessity of surgeries of SHPT (Fig. 3).

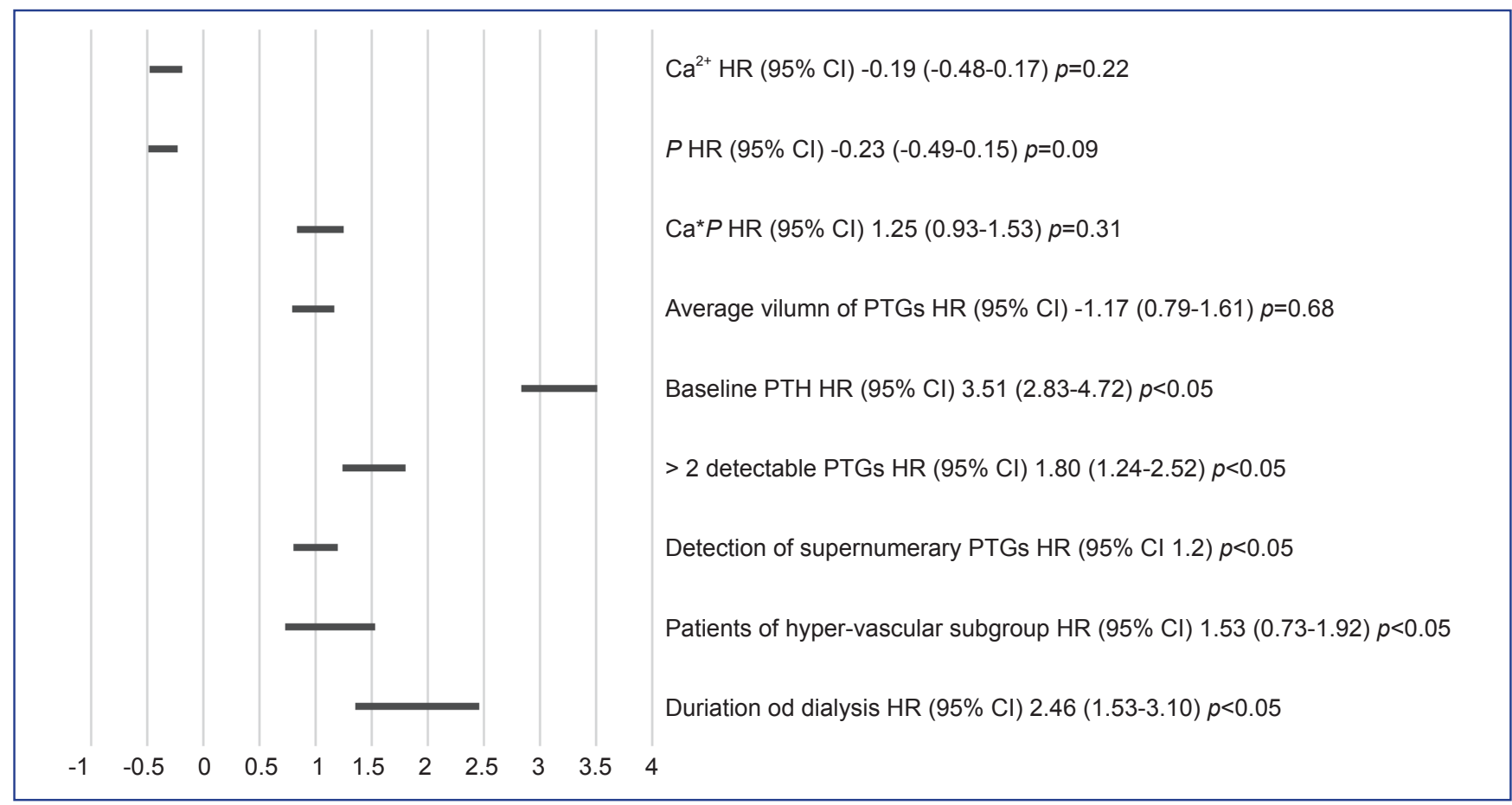

Figure 3 Multiple logistic regression analysis of the association between sonographic features, laboratory examinations and the necessity of surgeries.

In both groups, US detectable PTGs were more commonly seen in the left and right inferior regions than in the two superior regions, in spite of statistical insignificance $(p=0.58)$ (Fig. 4). The MLDs of PTGs in the four regions have no statistic difference either $(p=$ $0.20)$.

Surgical pathologies confirmed 121 PTGs, of which ten glands $(8.3 \%)$ were ectopic PTGs, including eight intra-thyroidal, one in carotid sheath, and one in submandibular region. Except for the one in submandibular region which was missed by US but detected by ${ }^{99 \mathrm{~m}} \mathrm{Tm}$-MIBI tomography, pre-operative US accurately distinguished 120 PTGs. On the other hand, six intra-thyroidal and 17 PTGs posterior to the thyroid gland were missed by $99 \mathrm{mTm}-\mathrm{MIBI}$ tomography. Hence, the detection rate of US was better than that of $99 \mathrm{mTm}$ MIBI tomography $(99.1 \%$ vs. $81.0 \%, p<0.01)$.
In the hyper-vascular subgroup, the average preoperative PTH was significantly higher than that in the hypo-vascular subgroup $(1219.5 \pm 783.1$ vs. $893.3 \pm$ $652.2 \mathrm{pmol} / \mathrm{L}, p<0.05$ ), while the pre-operative $\mathrm{Ca}^{2+}$ was significantly lower $(2.1 \pm 0.2$ vs. $2.4 \pm 0.2 \mathrm{mmol} /$ $\mathrm{L}, p<0.05$ ) in the hyper-vascular subgroup (Table 3 ). In correspondence with the result that the incidence of prolonged hypocalcemia was higher $(15.9 \%$ vs. $3.8 \%$, $p<0.05)$ in the hyper-vascular subgroup, the postoperative $\mathrm{Ca}^{2+}$ during the first $14 \mathrm{~d}$ after surgeries was also lower in the hyper-vascular subgroup (the average $\mathrm{Ca}^{2+}$ level, $2.0 \pm 0.1$ vs. $2.3 \pm 0.1 \mathrm{mmol} / \mathrm{L}, p<0.01$ ) (Fig. 5). The median follow-up time was 10 months (7-15 months) and 14 months (11-18 months) for patients in the surgical group and the medication group, respectively. Post-operative PTH levels in the surgical group declined to the KDIGO target range $(57-275 \mathrm{pg} / \mathrm{mL}$, average value 
$104.9 \pm 41.4 \mathrm{pg} / \mathrm{mL}$ ) [9], significantly lower than that in the medication group $(185-475 \mathrm{pg} / \mathrm{mL}$, average $226.9 \pm$ $143.4 \mathrm{pg} / \mathrm{mL}, p<0.05)$. During the 7-18 months follow- up period, one patient had mild bone pain, and one presented with itching in the medication group. Patients in neither group had severe clinical manifestations.

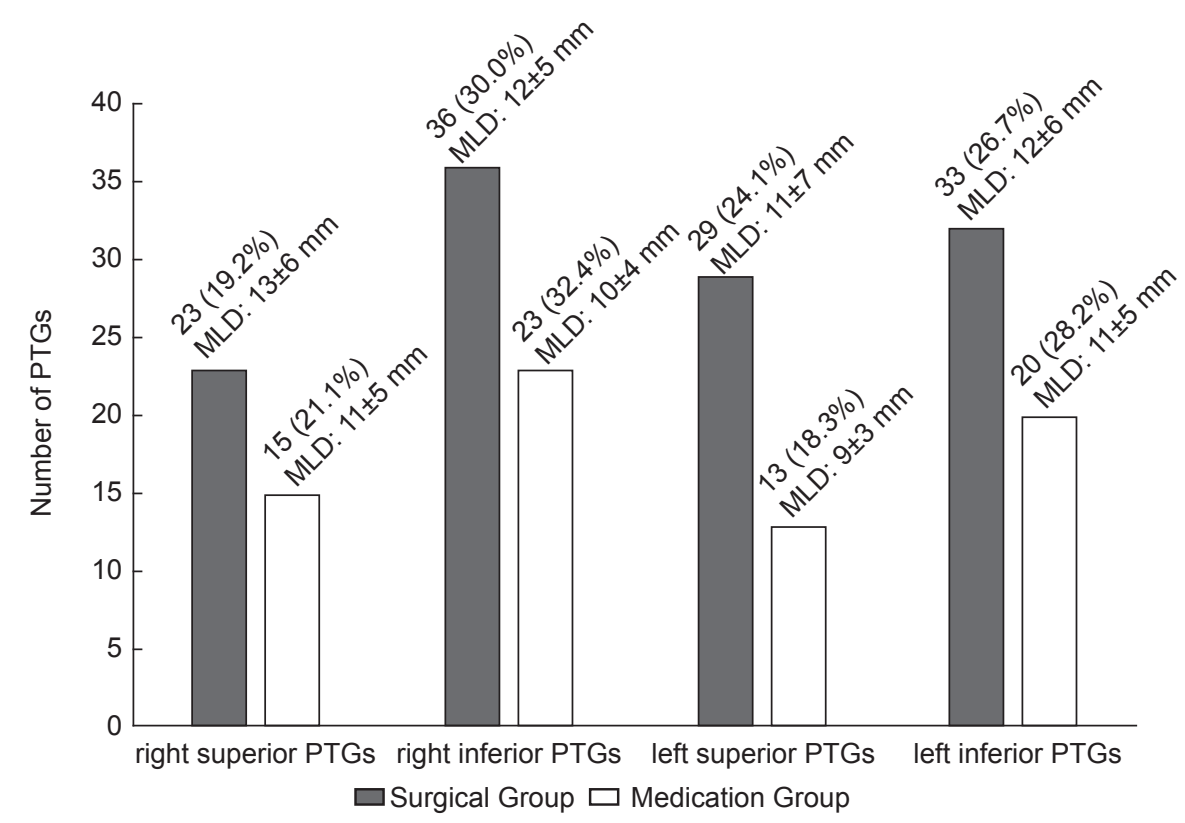

Figure 4 The distribution and maximal longitudinal diameters (MLDs) of US detectable parathyroid glands (PTGs) in surgical group and medication group.

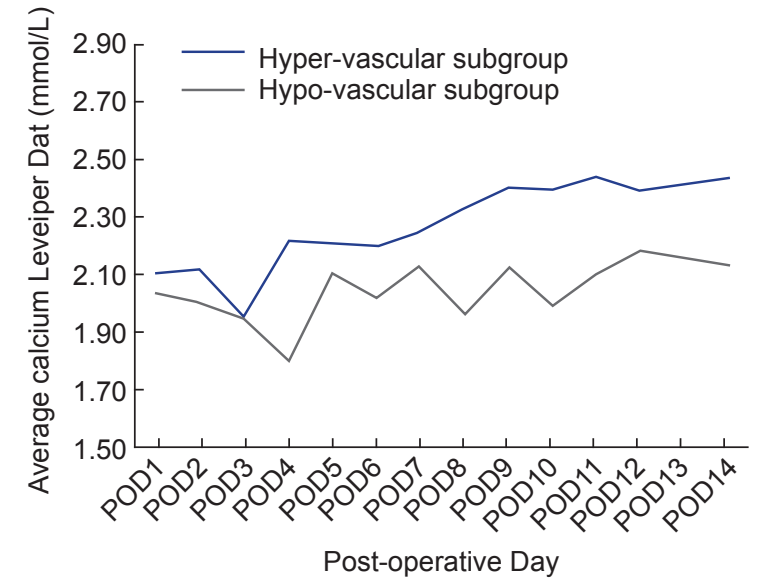

Figure 5 The average serum calcium $\left(\mathrm{Ca}^{2+}\right)$ monitoring curves from post-operative day 1 to day 14 for patients in hyper-vascular and hypovascular groups. According to the pre-operative sonographies, there were: (i) 22 cases in hyper-vascular subgroup, shown in gray line, (ii) 10 cases in hypo-vascular subgroup, shown in black line. The average serum Ca levels of patients in hyper-vascular subgroup was significantly lower than that in hypo-vascular subgroup $(2.0 \pm 0.1$ vs. $2.3 \pm 0.1, p<0.05)$.

\section{Discussion}

Associated with complications such as renal stones, osteoporosis, muscle weakness, itching, growth retardation, bone pain and cardiovascular events, SHPT is to blame for the substantial morbidity and mortality rate of patients on chronic hemodialysis. Approximately $40 \%-80 \%$ of SHPT patients with conservative treatments failed to maintain a PTH level to $<300 \mathrm{pg} / \mathrm{mL}[6,17]$.
For advanced SHPT, parathyroidectomy improves survival and cardiovascular outcomes $[18,19]$. In this study, medications were effective for $34.4 \%(22 / 64)$ of SHPT patients, when $15.6 \%(10 / 64)$ of patients were excluded because of dubious curative effect of medication. The rest $50 \%$ refractory to medication therapy had received surgical intervention in this patient study group. Ultrasonography of PTGs could be used to detect patients who are unlikely to respond to further medical therapy. But surgical indications based on sonographic findings for intervention were not conclusive. Additionally, the baseline and "cut-off" PTH levels of SHPT varied widely in reported studies [7-10]. Since mild patients more likely respond to pharmacological therapies [9], the present study focused on moderate and severe SHPT patients.

Compared to the medication group, the surgical group had a lower level of serum $\mathrm{Ca}^{2+}$ but a higher level of serum $\mathrm{P}$ and calcium-phosphorus product. These findings were similar to previous studies $[3,5]$. However, $\mathrm{Ca}^{2+}$, $\mathrm{P}$ and calcium-phosphorus product had no correlation with the patient response to drug therapy, this might be ascribed to the adequate control of serum electrolyte during six months of medication treatment. On the other hand, the baseline PTH $>2$ detectable PTGs, detection of supernumerary PTGs, patients of hyper-vascular subgroup, and the duration of dialysis were positively associated with the necessity of surgical intervention in SHPT treatment. 
Table 2 Demographics and clinical features of patients in the surgical group and medication group

\begin{tabular}{|c|c|c|c|}
\hline Items & Surgical group & Medication group & $P$ \\
\hline Age (years) & $47.7 \pm 12.8$ & $46.8 \pm 14.0$ & n.s \\
\hline Patients & 32 & 22 & \\
\hline
\end{tabular}

Sex

\begin{tabular}{|c|c|c|c|}
\hline Male & 20 & 7 & $\mathrm{n} . \mathrm{s}$ \\
\hline Female & 12 & 15 & \\
\hline Duration of dialysis (years) ${ }^{a}$ & $6.3 \pm 2.6$ & $5.4 \pm 3.5$ & $<0.05$ \\
\hline BUN $(\mu \mathrm{g} / \mathrm{L})$ & $13.5 \pm 7.4$ & $16.4 \pm 5.5$ & n.s. \\
\hline $\mathrm{Ca}^{2+}(\mathrm{mmol} / \mathrm{L})^{\mathrm{a}}$ & $2.3 \pm 0.5$ & $2.5 \pm 0.5$ & $<0.01$ \\
\hline $\mathrm{P}(\mathrm{mmol} / \mathrm{L})^{a}$ & $2.0 \pm 0.3$ & $1.7 \pm 0.5$ & $<0.05$ \\
\hline Baseline PTH $(\mathrm{pg} / \mathrm{mL})^{\mathrm{a}}$ & $924.5 \pm 781.7$ & $671.3 \pm 509.6$ & $<0.05$ \\
\hline $\mathrm{Ca}^{2+} * \mathrm{P}^{\mathrm{a}}$ & $4.6 \pm 2.2$ & $3.1 \pm 1.3$ & $<0.05$ \\
\hline Supernumerary PTGs locating posterior of the thyroid gland (num, \%) ${ }^{\text {a }}$ & $13(10.7 \%)$ & $2,2.8 \%$ & $<0.05$ \\
\hline Average volume of PTGs $\left(\mathrm{cm}^{3}\right)^{a}$ & $1.0 \pm 0.6$ & $0.9 \pm 0.7$ & $<0.05$ \\
\hline Total volume of PTGs $\left(\mathrm{cm}^{3}\right)$ & $2.7 \pm 1.9$ & $2.3 \pm 2.2$ & n.s \\
\hline US detectable PTGs (num, \%) & 120 & 71 & \\
\hline Homogeneous PTGs (num, \%) & $32,26.6 \%$ & $13,18.3 \%$ & n.s \\
\hline Hyper-echoic PTGs (num, \%) & $4,3.3 \%$ & $6,8.5 \%$ & \\
\hline Hypo-echoic PTGs (num, \%) & $73,60.8 \%$ & $47,66.2 \%$ & \\
\hline PTGs with calcification (num, \%) & $7,5.8 \%$ & $3,4.2 \%$ & \\
\hline \multicolumn{4}{|l|}{ Doppler score Onoda assement ${ }^{a}$} \\
\hline Patients in hyper-vascular subgroup (num, \%) & $18,56.3 \%$ & $10,45.4 \%$ & $<0.05$ \\
\hline Patients in hypo-vascular subgroup (num, \%) & $14,43.7 \%$ & $12,54.6 \%$ & \\
\hline
\end{tabular}

Scr, the serum creatinine; $\mathrm{Ca}^{2+}$, calcium; P, phosphorus; BUN, blood urea nitrogen; $\mathrm{Ca}$ *P, the calcium-phosphorus product; PTH, parathyroid hormone Variables are expressed as mean $\pm \mathrm{SD}$. Continuous variables were compared between groups using the $t$-test. Chi square test was used to compare and analyze the frequencies. a, the symbols indicate the difference $(p<0.05)$ between subgroups with respect to the higher value.

Table 3 Comparison between hyper- and hypo-vascular PTGs in the surgical group

\begin{tabular}{|c|c|c|c|c|c|c|}
\hline $\begin{array}{l}\text { Surgical group } \\
\quad(n=32)\end{array}$ & $\begin{array}{l}\text { Numbers of } \\
\text { excised PTGs }\end{array}$ & $\begin{array}{l}\text { Average volume of } \\
\text { excised PTGs of } \\
\text { each patient }\left(\mathrm{cm}^{3}\right)\end{array}$ & $\begin{array}{l}\text { Average pre- } \\
\text { operative PTH } \\
\quad(\mathrm{pmol} / \mathrm{L})\end{array}$ & $\begin{array}{l}\text { Average pre- } \\
\text { operative } \mathrm{Ca}^{2+} \\
(\mathrm{mmol} / \mathrm{L})\end{array}$ & $\begin{array}{l}\text { Patients with } \\
\text { prolonged } \\
\text { hypocalcemia } \\
\quad(n=15)\end{array}$ & $\begin{array}{c}\text { Average post- } \\
\text { operative } \mathrm{Ca}^{2+} \text { in } 14 \\
\text { days } \\
(\mathrm{mmol} / \mathrm{L})\end{array}$ \\
\hline $\begin{array}{l}\text { Hyper-vascular } \\
\text { subgroup }(n=18)\end{array}$ & 69 & $3.7 \pm 1.8$ & $1219.5 \pm 783.1^{\mathrm{a}}$ & $2.1 \pm 0.5^{\mathrm{a}}$ & $11(15.9 \%)^{a}$ & $2.0 \pm 0.7^{\mathrm{a}}$ \\
\hline $\begin{array}{c}\text { Hypo-vascular } \\
\text { subgroup }(n=14)\end{array}$ & 52 & $4.0 \pm 2.1$ & $893.3 \pm 652.2^{a}$ & $2.4 \pm 0.4^{\mathrm{a}}$ & $2(3.8 \%)^{a}$ & $2.3 \pm 0.6^{\mathrm{a}}$ \\
\hline
\end{tabular}

$\mathrm{Ca}$, calcium; PTH, parathyroid hormone. Variables are expressed as mean $\pm \mathrm{SD}$. Continuous variables were compared between groups using the $t$-test. Chi square test was used to compare and analyze the frequencies. a, the symbols indicate the difference $(p<0.05)$ between subgroups with respect to the higher value. 
Most current researches emphasized the laboratory measurements, but few explored the ultrasonic features of SHPT. In this study, it was first proposed that, US was effective and essential to detect the number and the vascularity of PTGs, it could help clinicians to determine surgical intervention for moderate and severe SHPT patients refractory to medical management.

In the surgical group, only 50\% patients had 4 PTGs, much lower than that reported previously [20]. The detection of supernumerary PTGs was significantly higher in the surgical group than that in the medication group ( $p<$ $0.05)$. There was a medical hypothesis "seed, environment, and soil" proposing that, during embryonic development, multiple "micro-parathyroids" may become potential auxiliary sources secreting PTH. In patients undergoing dialysis, various physiological stimuli may result in the activation of micro-parathyroids, even the development of "newborn" supernumerary PTGs [21-23]. However, the "micro-parathyroids" might only be recognized by postoperative pathologies rather than the surgeons. Thus, some experts proposed a novel surgical strategy for SHPT, namely purge parathyroidectomy (PPTX), to remove all PTGs posterior to the thyroid gland and the potential supernumerary or ectopic parathyroid tissues, the thymus tongue, and the cervical fibro-fatty tissues through the routine curved neck incision [21]. Our opinion is that the US detection of supernumerary PTGs posterior to the thyroid gland might predict the necessity of surgical intervention.

Patients with SHPT were considered as not to have symmetric enlargement of the PTGs [24]. Instead, the MLD of PTGs presented in the four typical regions had no statistic difference in this study. For moderate and severe patients with PTH $>500 \mathrm{pg} / \mathrm{mL}$, volumetric changes of PTGs might be symmetric. Detectable PTGs in bilateral inferior positions were more common than that in superior positions. This could be attributed to the relative independent blood supply of inferior PTGs than that of superior ones, which made the glands easier to be traced and identified by US.

US findings appeared to be related to the serum $\mathrm{Ca}^{2+}$ level after operation within a short timeframe. Patients in the hyper-vascularity subgroup were more likely to have severe hypocalcemia despite receiving standard postoperative supportive treatment. Hypervascularity might implicate supplementing adequate nutrition to maintain the hyper-function of PTGs. Hence, serum $\mathrm{Ca}^{2+}$, which was regulated by PTGs, would decrease sharply after removing the abundant blood supply to the PTGs. Previous studies only revealed age, preoperative $\mathrm{PTH}$ and $\mathrm{Ca}^{2+}$ level as risk factors of severe hypocalcemia after PXT. This study presented that patients with hyper-vascularity of the PTGs dominated $(\geqslant 50 \%)$ was correlated with increased risk of severe hypocalcemia after PXT. US might help screen highrisk patients of severe hypocalcemia, and implement preventative calcium dialysate and large doses of $\mathrm{Ca}^{2+}$ supplementation after surgeries.

There are some limitations of this study. Certainly, larger sample sizes and a longer follow-up period are required to fully determine the efficacy of US features to help the management of SHPT.

\section{Conclusion}

For moderate and sever SHPT patients, US features can provide useful information for surgical intervention of SHPT and predict the risk of prolonged hypocalcemia after PXT, which might optimize clinical managements, such as implementation of surgical intervention, supplementation of preventative $\mathrm{Ca}^{2+}$ dialysate and large doses of $\mathrm{Ca}^{2+}$ postoperatively.

\section{Acknowledgments}

We would like to thank Dr. Yu Tang and Dr. Wenli Hou for helping language editing.

\section{Conflict of Interest}

We declare that we have no financial and personal relationships with other people or organizations that can inappropriately influence our work, there is no professional or other personal interest of any nature or kind in any product, service and/or company that could be construed as influencing the position presented in, or the review of the manuscript.

\section{References}

[1] Katoh N, Nakayama M, Shigematsu T, Yamamoto H, Sano K, Saito I, et al. Presence of sonographically detectable parathyroid glands can predict resistance to oral pulsed-dose calcitriol treatment of secondary hyperparathyroidism. Am J Kidney Dis 2000; 35: 465-468.

[2] Tominaga Y, Matsuoka S, Sato T, Uno N, Goto N, Katayama A, et al. Clinical features and hyperplastic patterns of parathyroid glands in hemodialysis patients with advanced secondary hyperparathyroidism refractory to maxacalcitol treatment and required parathyroidectomy. Ther Apher Dial 2007; 11: 266-273.

[3] Young EW, Albert JM, Satayathum S, Goodkin DA, Pisoni RL, Akiba T, et al. Predictors and consequences of altered mineral metabolism: the Dialysis Outcomes and Practice Patterns Study. Kidney Int 2005; 67: 1179-1187.

[4] Onoda N, Kurihara S, Sakurai Y, Owada, K, Osono, E, Adachi, H, et al. Evaluation of blood supply of the parathyroid glands in secondary hyperparathyroidism compared to histopathology. Nephrol Dial Transplant 2003;18: 34-37

[5] van der Plas WY, Engelsman AF, Özyilmaz A, van der HorstSchrivers AN, Meijer K, van Dam GM, et al. Impact of the Introduction of calcimimetics on timing of parathyroidectomy in secondary and tertiary hyperparathyroidism. Ann Surg Oncol 2017; 24: 15-22.

[6] Vulpio C, Bossola M, De Gaetano A, Maresca G, Di Stasio E, Spada PL, et al. Ultrasound patterns of parathyroid glands in chronic 
hemodialysis patients with secondary hyperparathyroidism. Am J Nephrol 2008; 28: 589-597.

[7] Fukagawa M, Nakanishi S, Kazama JJ. Basic and clinical aspects of parathyroid hyperplasia in chronic kidney disease. Kidney Int Suppl 2006; 102: S3-7.

[8] Fukagawa M, Kitaoka M, Yi H, Fukuda N, Matsumoto T, Ogata E, et al. Serial evaluation of parathyroid size by ultrasonography is another useful marker for the long-term prognosis of calcitriol pulse therapy in chronic dialysis patient. Nephron 1994; 68: 221-228.

[9] National Kidney Foundation. KDOQI clinical practice guidelines for bone metabolism and disease in chronic kidney disease. Am J Kidney Dis 2003; 42:1-201.

[10] Vulpio C, Bossola M. Parathyroid nodular hyperplasia and responsiveness to drug therapy in renal secondary hyperparathyroidism: an open question. Therapeutic Apheresis and Dialysis 2018; 22:11-21.

[11] Latus J, Roesel M, Fritz P, Braun N, Ulmer C, Steurer W, et al. Incidence of and risk factors for hungry bone syndrome in 84 patients with secondary hyperparathyroidism. Int J Nephrol Renov Dis 2013; 6:131-137.

[12] Goldfarb M, Gondek SS, Lim SM, Farra JC, Nose V, Lew JI. Postoperative hungry bone syndrome in patients with secondary hyperparathyroidism of renal origin. World J Surg 2012; 36:13141319

[13] Fang L, Tang B, Hou D, Meng M, Xiong M, Yang J. Relationship between parathyroid mass and parathyroid hormone level in hemodialysis patients with secondary hyperparathyroidism. BMC Nephrol 2015; 16: 82.

[14] Madorin C, Owen RP, Fraser WD, Pellitteri PK, Radbill B, Rinaldo A, et al. The surgical management of renal hyper-parathyroidism. Eur Arch Otorhinolaryngol 2012; 269:1565-1576.

[15] Schneider R, Bartsch DK. Role of surgery in the treatment of renal secondary hyperparathyroidism. Br J Surg 2015; 102: 289-290.

[16] Ho LY, Wong PN, Sin HK, Wong YY, Lo KC, Chan SF, et al. Risk factors and clinical course of hungry bone syndrome after total parathyroidectomy in dialysis patients with secondary hyperparathyroidism. BMC Nephrol 2017;18:12.

[17] Goldsmith D, Covic A, Vervloet M, Cozzolino M, Nistor I. Should patients with $\mathrm{CKD}$ stage 5D and biochemical evidence of secondary hyperparathyroidism be prescribed calcimimetic therapy? an era-edta position statement. Nephrol Dial Transplant 2015; 30:698-700.

[18] Komaba H, Taniguchi M, Wada A, Iseki K, Tsubakihara Y, Fukagawa M. Parathyroidectomy and survival among Japanese hemodialysis patients with secondary hyperparathyroidism. Kidney Int 2015; 88:350-359.

[19] Iwamoto N, Sato N, Nishida M, Hashimoto T, Kobayashi H, Yamasaki S, et al. Total parathyroidectomy improves survival of hemodialysis patients with secondary hyperparathyroidism. $J$ Nephrol 2012; 25:755-763.

[20] Andrade JS, Mangussi-Gomes JP, Rocha LA, Ohe MN, Rosano M, das Neves MC, et al. Localization of ectopic and supernumerary parathyroid glands in patients with secondary and tertiary hyperparathyroidism: surgical description and correlation with preoperative ultrasonography and Tc99m-Sestamibi scintigraphy. Braz J Otorhinolaryngol 2014; 80: 29-34

[21] Alkhalili E, Tasci Y, Aksoy E, Aliyev S, Soundararajan S, Taskin E, et al. The utility of neck ultrasound and sestamibi scans in patients with secondary and tertiary hyperparathyroidism. World J Surg 2015; 39: 701-705.

[22] Wagner PK, Seesko HG, Rothmund M. Replantation of cryopreserved human parathyroid tissue. World J Surg 1991; 15: 751755.

[23] Wang Q, Palnitkar S, Parfitt AM. The basal rate of cell proliferation in normal human parathyroid tissue: implications for the pathogenesis of hyperparathyroidism. Clin Endocrinol 1997; 46: 343-349.

[24] Takebayashi S, Matsui K, Onohara Y, Hidai H. Sonography for early diagnosis of enlarged parathyroid glands in patients with secondary hyperparathyroidism. AJR Am J Roentgenol 1987; 148:911-914. 